\title{
Depressão Materna e Interação Mãe-Bebê no Final do Primeiro Ano de Vida ${ }^{1}$
}

\author{
Daniela Delias de Sousa Schwengber \\ Cesar Augusto Piccinini ${ }^{2}$ \\ Universidade Federal do Rio Grande do Sul
}

\begin{abstract}
RESUMO - O objetivo deste estudo foi o de examinar eventuais diferenças na interação mãe-bebê entre mães com e sem depressão no final do primeiro ano de vida do bebê. Participaram 26 díades mãe-bebê, 11 com mães com indicadores de depressão e 15 com mães sem indicadores. A designação aos dois grupos ocorreu com base nos escores do Inventário Beck de Depressão. Análise dos totais de comportamentos maternos e infantis durante sessão de observação do brinquedo livre revelou que mães com indicadores de depressão apresentaram menos comportamentos facilitadores da exploração de brinquedos pelos bebês enquanto seus filhos mostraram mais afeto negativo. Além disso, mães com indicadores de depressão evidenciaram mais apatia, mantiveram menos a atenção de seus filhos nos brinquedos e demonstraram menos ternura e afeição e seus bebês apresentaram mais vocalizações negativas. Esses resultados apóiam as expectativas de que a depressão materna pode ocasionar um impacto negativo na interação mãe-bebê.
\end{abstract}

Palavras-chave: depressão materna; interação mãe-bebê.

\section{Maternal Depression and Mother-Infant Interaction by the End of the First Year of Life}

\begin{abstract}
The aim of this study was to examine eventual differences in mother-infant interaction between mothers with and without indicators of depression by the end of the first year of the baby's life. Twenty-six mother-infant dyads, eleven comprising mothers with indicators of depression and fifteen with mothers without indicators, took part in the study. The assignment to both groups was based on scores on the Beck Depression Inventory. Analysis of the total of maternal and infant behaviours during a free-play observation session revealed that mothers with indicators of depression presented less behaviours that facilitate exploration of toys by the infant, and their children presented more negative affect. Furthermore, mothers with indicators of depression showed more apathy, kept less their children's attention in the toys, demonstrated less tenderness and affection, and their babies presented more negative vocalizations. These results give support to the expectations that maternal depression may produce a negative impact on mother-infant interaction.
\end{abstract}

Key words: maternal depression; mother-infant interaction.

O primeiro ano após o nascimento de um filho representa um período propício para a ocorrência de sintomas de depressão entre as mulheres (Klaus, Kennell \& Klaus, 2000). O papel exercido pela depressão materna na interação mãe-bebê tem sido objeto de estudo de inúmeras investigações nas últimas décadas, devido a evidências de que o estado depressivo da mãe pode repercutir negativamente no estabelecimento das primeiras interações com o bebê e, em conseqüência, no desenvolvimento afetivo, social e cognitivo da criança (Cummings \& Davies, 1994; Dodge, 1990; Field, 1998; Tronick \& Weinberg, 1997). Porém, o exame da literatura revela que grande parte dos estudos que versam sobre a interação bebê-mãe deprimida abordou as implicações da depressão materna para a interação nos primeiros meses após o nascimento da criança, em virtude da maior incidência dos quadros depressivos nesse período (Cox, Murray \& Chapman, 1993; O’Hara, Neunaber

1 Artigo baseado na Dissertação de Mestrado da primeira autora, desenvolvida sob orientação do segundo autor.

2 Endereço: Universidade Federal do Rio Grande do Sul, Rua Ramiro Barcelos, 2600/111, Porto Alegre, RS, Brasil 90035-006. E-mail: piccinini@portoweb.com.br
\& Zekoski, 1984). Nesse sentido, alguns autores chamaram a atenção para a necessidade da realização de investigações após esse período inicial, ao sugerirem que algumas mulheres permanecem com os sintomas por um período prolongado enquanto outras começam a se sentir deprimidas mais tardiamente no primeiro ano após o parto (Beck \& Steer, 1993; Brown, Lumley, Small \& Astbury, 1994; Klaus \& cols., 2000; Murray, Cox, Chapman \& Jones, 1995). Da mesma forma, salientaram a importância de se considerar que os efeitos da depressão materna para a interação mãe-bebê estão associados ao tempo de permanência dos sintomas e à cronicidade do quadro depressivo (Beeghly \& cols. 2002; Campbell \& Cohn, 1997; Campbell, Cohn \& Meyers, 1995; Cooper, Campbell, Day, Kennerley \& Bond, 1988).

Os estudos que avaliaram o impacto da depressão materna para a interação mãe-bebê em períodos mais avançados do desenvolvimento do bebê não utilizaram observações da interação face-a-face, mas, sim, situações de jogo nas quais as habilidades e comportamentos mais sofisticados da criança pudessem ser observados (Schwengber \& Piccinini, 2001). Alguns desses estudos examinaram os comportamentos maternos e infantis durante a exploração de brinquedos 
pelos bebês em situações de jogo com suas mães. De acordo com Lawson, Parrinello e Ruff (1992) e Tamis-LeMonda e Bornstein (1989), essa exploração por parte do bebê durante a interação com a mãe estaria associada ao seu desenvolvimento cognitivo posterior. Dessa forma, comportamentos maternos de introduzir brinquedos e manter a atenção do bebê em um brinquedo pelo qual mostrou interesse estariam associados a um aumento na exploração de objetos por parte do bebê enquanto que o redirecionamento de sua atenção e a manifestação de intrusividade por parte da mãe estariam relacionados a um decréscimo nessa exploração.

Em um desses estudos, Hart, Field, Del Valle e PelaezNogueras (1998) encontraram que os bebês de mães deprimidas mostraram-se menos engajados na exploração de objetos e que filhas de mães deprimidas apresentaram mais afeto negativo. Além disso, mães deprimidas cujos bebês eram meninos foram mais intrusivas do que mães nãodeprimidas. Embora não tenham sido encontradas diferenças entre os grupos quanto à introdução de brinquedos, mães deprimidas introduziram mais brinquedos para meninos do que para meninas. Esses resultados corroboraram evidências a respeito da maior intrusividade e envolvimento ativo na interação das mães deprimidas com meninos (Radke-Yarrow $\&$ cols., 1995).

Ainda em relação à questão da intrusividade, um estudo posterior realizado por Hart, Jones, Field e Lundy (1999) revelou a existência de dois estilos distintos que uma mãe deprimida pode desenvolver em interação com seu filho: o primeiro é caracterizado pelo afastamento, falta de engajamento e pouca estimulação; o segundo caracteriza-se por comportamentos intrusivos e de superestimulação. Esse estudo revelou que mães deprimidas intrusivas apresentaram mais respostas positivas, mais demonstração de brinquedos e uma tendência maior a guiarem fisicamente seus bebês que, no entanto, mostraram menos manipulação de objeto. Por outro lado, mães deprimidas que se mostraram mais apáticas, quietas e afastadas mantiveram a criança brincando com mais freqüência e mostraram afeto mais restrito, enquanto suas crianças demonstraram menos expressão afetiva, positiva ou não.

Os resultados referentes à apatia evidenciada por mães deprimidas corroboram aqueles encontrados por Seiner e Gelfand (1995), ao avaliarem o efeito da simulação materna de depressão e afastamento para a interação mãe-bebê. Diante das mães menos responsivas, expressivas, envolvidas e falantes, os bebês tendiam a se afastar fisicamente e apresentavam mais comportamentos negativos para chamar atenção. Quando as mães não estavam simulando afeto depressivo e afastamento, os bebês evidenciavam mais comportamentos positivos e brincavam mais próximos às mães.

Além dos estilos interativos apresentados por mães deprimidas, as expressões afetivas das díades durante a interação mãe-bebê também foram investigadas em alguns estudos sobre o impacto da depressão da mãe em estágios mais avançados do desenvolvimento do bebê. Em um desses estudos, Radke-Yarrow, Nottelmann, Belmont e Welsh (1993) examinaram diferenças na ocorrência de algumas expressões de afeto de mães deprimidas e não-deprimidas e de seus bebês. Os resultados indicaram que mães deprimidas mostraram mais afeto negativo do que mães não-deprimidas e apontaram também para uma correlação entre o afeto das mães e dos bebês.

Considerando o exposto, o presente estudo buscou ampliar as investigações relatadas na literatura, examinando as implicações da depressão materna na interação mãe-bebê aos 12 meses de vida, em uma situação de brinquedo livre. Com base na literatura, a expectativa inicial era de que mães com indicadores de depressão tenderiam a apresentar menos comportamentos facilitadores da exploração de brinquedos pelo bebê, como introdução de brinquedos, manutenção da atenção do bebê nos brinquedos e demonstração física e verbal de afeto, e mais comportamentos não-facilitadores dessa exploração, como redirecionamento da atenção dos bebês para outros brinquedos, intrusividade, contrariedade e apatia. Além disso, esperava-se que os bebês de mães com indicadores de depressão demonstrassem mais afeto negativo (rejeição de brinquedos, choro, vocalizações negativas e afastamento da mãe) do que os bebês de mães sem indicadores de depressão, e menos afeto positivo (definido este como focalização da atenção nos brinquedos, sorrisos, vocalizações positivas e busca de proximidade da mãe).

\section{Método}

\section{Participantes}

Participaram deste estudo 26 díades mãe-bebê, 11 das quais com mães com indicadores de depressão (moderada: 4; leve: 7) e 15 com mães sem indicadores de depressão. A designação aos dois grupos ocorreu a partir dos escores obtidos pelas mães nas respostas ao Inventário Beck de Depressão (Beck \& Steer, 1993), quando o bebê estava com 12 meses. Todas as mães eram primíparas, não apresentaram complicações físicas durante a gestação e o parto e, com exceção de uma mãe em cada grupo, viviam com os pais dos seus bebês. Os bebês eram de ambos os sexos, com a idade de 12 meses por ocasião da investigação.

A amostra foi selecionada, com base nos critérios descritos acima, dentre os participantes do "Estudo Longitudinal de Porto Alegre: Da Gestação à Escola” (Piccinini, Tudge, Lopes \& Sperb, 1998). Este estudo iniciou acompanhando aproximadamente 100 famílias, cujas gestantes eram primíparas, representando várias configurações familiares, de diferentes idades, escolaridade e níveis socioeconômicos. $\mathrm{O}$ estudo buscou atender todas as normas éticas de conduta em pesquisa com seres humanos, tendo sido aprovado pelo Comitê de Ética do Hospital de Clínicas de Porto Alegre (Proc. $\mathrm{n}^{\circ}$ 98293). O contato inicial para participar deste estudo foi feito com a gestante no terceiro trimestre de gestação, através de hospitais da rede pública e privada de Porto Alegre, postos de saúde, por indicação e anúncios em jornais. Quando os bebês tinham três, oito e doze meses foram feitas coletas de dados sobre seu desenvolvimento e interações familiares. Para o presente estudo foram utilizados os dados obtidos aos doze meses de vida do bebê. Detalhes de todas as coletas de dados são descritos por Piccinini e cols. (1998).

Dentre os participantes do presente estudo, 14 foram selecionados em hospitais, dois em postos de saúde, seis por indicação e quatro através de divulgação na mídia. As Tabelas 1 e 2 apresentam as características demográficas 
Tabela 1. Dados demográficos das mães com indicadores de depressão

\begin{tabular}{rlcccccc}
\hline $\mathbf{N}^{\mathbf{0}}$ & Nível de Depressão & Escore BDI & Idade & Estado civil & $\begin{array}{c}\text { Escolaridade } \\
\text { em anos }\end{array}$ & Ocupação & Sexo do bebê \\
\hline 1 & Moderada & 31 & 19 & Solteira & 11 & Estudante & Masculino \\
2 & Moderada & 20 & 20 & Casada & 10 & Não trabalha fora & Masculino \\
3 & Moderada & 20 & 20 & Casada & 8 & Babá & Masculino \\
4 & Moderada & 20 & 24 & Casada & 8 & Não trabalha fora & Feminino \\
5 & Leve & 16 & 27 & Casada & 11 & Não trabalha fora & Feminino \\
6 & Leve & 16 & 18 & Casada & 10 & Estudante & Feminino \\
7 & Leve & 15 & 24 & Casada & 6 & Confeiteira & Masculino \\
8 & Leve & 15 & 20 & Casada & 11 & Comerciante & Masculino \\
9 & Leve & 15 & 20 & Casada & 11 & Aux. Escritório & Masculino \\
10 & Leve & 12 & 15 & Casada & 8 & Estudante & Feminino \\
11 & Leve & 12 & 24 & Casada & 10 & Garçonete & Feminino \\
\hline
\end{tabular}

Tabela 2. Dados demográficos das mães sem indicadores de depressão

\begin{tabular}{llrrlrll}
\hline $\mathbf{N}^{\mathbf{0}}$ & Nível de depressão & Escore BDI & Idade & Estado civil & $\begin{array}{c}\text { Escolaridade } \\
\text { em anos }\end{array}$ & Ocupação & Sexo do bebê \\
\hline 12 & Ausente & 11 & 23 & Casada & 11 & Vendedora & Feminino \\
13 & Ausente & 11 & 18 & Casada & 9 & Não trabalha fora & Masculino \\
14 & Ausente & 11 & 28 & Casada & 13 & Não trabalha fora & Masculino \\
15 & Ausente & 10 & 32 & Casada & 8 & Aux. Lavanderia & Masculino \\
16 & Ausente & 10 & 26 & Casada & 13 & Não trabalha fora & Feminino \\
17 & Ausente & 9 & 28 & Casada & 11 & Serviços gerais & Masculino \\
18 & Ausente & 8 & 18 & Solteira & 11 & Estudante & Masculino \\
19 & Ausente & 8 & 19 & Casada & 7 & Doméstica & Masculino \\
20 & Ausente & 7 & 15 & Casada & 8 & Não trabalha fora & Feminino \\
21 & Ausente & 6 & 33 & Casada & 15 & Fonoaudióloga & Feminino \\
22 & Ausente & 6 & 31 & Casada & 16 & Programadora & Feminino \\
23 & Ausente & 6 & 27 & Casada & 4 & Não trabalha fora & Masculino \\
24 & Ausente & 6 & 25 & Casada & 8 & Não trabalha fora & Masculino \\
25 & Ausente & 6 & 32 & Casada & 17 & Cons. Familiar & Masculino \\
26 & Ausente & 4 & 24 & Casada & 11 & Não trabalha fora & Masculino \\
\hline
\end{tabular}

dos participantes. No grupo de mães com indicadores de depressão as idades variaram entre 15 e 27 anos $(M=21$; $D P=3,4)$ e no grupo de mães sem indicadores de depressão entre 15 e 33 anos $(M=25,3 ; D P=5,7)$. A escolaridade no primeiro grupo variou de seis a $11 \operatorname{anos}(M=9,4 ; D P=1,7)$ e no segundo de quatro a 17 anos $(M=10,8 ; D P=3,6)$. O nível socioeconômico variou de baixo a alto, com base na escolaridade e profissão das mães. Às mães cujos escores obtidos no $\mathrm{BDI}^{3}$ revelaram a presença de indicadores de depressão foi oferecido atendimento psicoterápico por terapeutas integrantes do grupo de pesquisa, após a coleta de dados quando se verificou a depressão.

\section{Delineamento e procedimentos}

Foi utilizado um delineamento de grupos contrastantes (Nachmias \& Nachmias, 1996), sendo um grupo com díades cujas mães apresentavam indicadores de depressão e o outro

3 O termo "BDI" refere-se ao Inventário Beck de Depressão. De acordo com as normas da versão em português (Cunha, 2001), o nível de depressão é classificado em mínimo (0 a 11 pontos), leve (12 a 19 pontos), moderado ( 20 a 35 pontos) e grave ( 36 a 63 pontos). com díades cujas mães não apresentavam indicadores de depressão. Examinou-se a interação entre as díades dos dois grupos aos 12 meses de vida do bebê, particularmente no que se refere ao seu engajamento na exploração de brinquedos. As famílias eram contatadas e convidadas para compareceram à Sala de Brinquedos do Instituto de Psicologia da UFRGS, quando era realizada a Observação da Interação Familiar, e a mãe respondia ao Inventário Beck de Depressão (Beck \& Steer, 1993). Nesta ocasião outros instrumentos também foram aplicados, cujos detalhes encontram-se em Piccinini e cols. (1998), mas que não são analisados no presente estudo.

\section{Instrumentos}

Observação da Interação Familiar (GIDEP, 1998): esta sessão de observação constava de uma seqüência de quatro episódios de interação livre entre a família: pai-mãe-bebê, pai-bebê, mãe-bebê e novamente pai-mãe-bebê. Cada sequiência tinha a duração de oito minutos e foram filmadas. Para o presente estudo, analisou-se seis minutos da interação livre do bebê com a mãe. O primeiro minuto foi descartado por ser um período de adaptação ao novo contexto. O último minuto foi descartado tendo em vista que em alguns casos a filmagem foi 
concluída ainda dentro do oitavo minuto, o que impossibilitou que fosse utilizado completamente. Para fins de análise, os seis minutos utilizados foram divididos em intervalos de 15 segundos, totalizando 24 intervalos. Em cada intervalo examinou-se a presença de oito categorias de comportamentos maternos e oito categorias de comportamentos do bebê, de acordo com um protocolo desenvolvido por Schwengber e Piccinini (2001), o qual foi baseado nos sistemas de codificação originalmente desenvolvidos por Hart e cols. (1998), Lawson e cols. (1992) e Radke-Yarrow e cols. (1993). As categorias de comportamentos maternos foram: introduz um brinquedo, mantém a atenção em um brinquedo, redireciona a atenção para outro brinquedo, evidencia intrusividade ao brincar, expressa prazer/alegria, demonstra ternura/afeição, evidencia apatia, demonstra contrariedade. As categorias de comportamentos do bebê foram: focaliza a atenção em um brinquedo, rejeita um brinquedo, sorri, chora, vocaliza positivamente, vocaliza negativamente, busca proximidade, afasta-se/resiste ao contato.

Dois observadores foram treinados para a utilização do protocolo de observação durante aproximadamente 30 horas, assistindo a vídeos de interação mãe-bebê de outros participantes do projeto de pesquisa longitudinal. O treinamento levou a algumas modificações na definição das categorias de comportamentos para melhor adequação do instrumento. Após esse treinamento inicial, seis casos foram utilizados pelos dois codificadores para o cálculo do índice de concordância na análise das categorias, realizado através do Kappa. Nas categorias de comportamentos maternos o índice de concordância atingiu $0,67^{4}$ enquanto que nas categorias de comportamentos do bebê atingiu 0,85 . Uma vez estabelecidos esses índices, os dois codificadores examinaram separadamente todos os outros 20 videoteipes. Eventuais diferenças entre os codificadores foram dirimidas posteriormente por um terceiro codificador, no caso a pesquisadora.

Inventário Beck de Depressão - BDI (Beck \& Steer, 1993): este instrumento é uma escala sintomática de autorelato, composta por 21 itens com diferentes alternativas de resposta a respeito de como o sujeito tem se sentido recentemente, e que correspondem a diferentes níveis de gravidade da depressão. A soma dos escores dos itens individuais fornece um escore total, que por sua vez constitui um escore dimensional da intensidade da depressão, que pode ser classificado nos seguintes níveis: mínimo, leve, moderado ou grave. A versão em português do Inventário Beck de Depressão resultou de uma formulação consensual da tradução do original em inglês, com a colaboração de quatro psicólogos clínicos, quatro psiquiatras e uma tradutora, sendo testada junto com a versão em inglês em 32 pessoas bilíngües, com três dias de intervalo e variando a ordem da apresentação dos dois idiomas nas duas metades da amostra (Cunha, 2001; Cunha, Prieb, Goulart \& Lemes, 1996). A consistência interna do BDI foi de 0,84 e a correlação entre teste e reteste foi de $0,95(p<0,001)$. Tendo em vista que muitas das participantes do presente estudo possuíam nível educacional baixo, optou-se por aplicar separadamente cada

4 De acordo com Robson (1995), os valores do Kappa são assim classificados: suficientes $(0,40$ a 0,60$)$; bons $(0,60$ a 0.75$)$; e excelentes (acima de 0,75$)$. item do inventário, os quais foram apresentados em cartões pelos pesquisadores para cada participante. Um exame inicial da aplicação mostrou que esse procedimento facilitava a compreensão por parte das participantes.

\section{Resultados}

Uma análise inicial dos dados foi realizada para examinar o efeito geral da condição materna (com e sem indicadores de depressão) nos escores totais de comportamentos maternos e infantis. Para tanto, as categorias maternas foram agrupadas e denominadas facilitadoras ou não-facilitadoras da interação mãe-bebê, em particular no engajamento do bebê na exploração de brinquedos. As categorias classificadas como facilitadoras foram: introduz um brinquedo, mantém a atenção em um brinquedo, expressa prazer e alegria e demonstra ternura e afeição. Já as categorias classificadas como não-facilitadoras foram: redireciona a atenção para outro brinquedo, evidencia intrusividade ao brincar, mostra apatia e mostra contrariedade. Da mesma forma, as categorias de comportamentos infantis foram agrupadas e denominadas de afeto positivo e afeto negativo. As categorias compreendidas como envolvendo afeto positivo foram: focaliza a atenção em um brinquedo, sorri, vocaliza positivamente e busca proximidade. As categorias consideradas afeto negativo foram: rejeita um brinquedo, chora, vocaliza negativamente e afasta-se/resiste ao contato.

A Tabela 3 apresenta a incidência média, desvio padrão, valor de F e nível de significância para os escores totais de comportamentos maternos e infantis. Para examinar o efeito geral da presença de indicadores de depressão materna nos escores totais de comportamentos maternos e infantis, duas análises multivariadas (MANOVA) foram realizadas separadamente, sendo uma para os escores totais de comportamentos maternos e outra para os escores totais de comportamentos infantis. Uma análise inicial não demonstrou nenhum efeito do sexo do bebê, idade e escolaridade da mãe na incidência total de comportamentos maternos e infantis. Por essa razão, esses fatores não foram utilizados como co-variantes nas análises multivariadas. Procedimento similar foi adotado por Hossain, Field, Gonzalez, Malphurs e Del Valle (1994), PelaezNogueras, Field, Cigales, Gonzalez e Clasky (1994) e Hart e cols. (1999) em estudos envolvendo depressão materna que utilizaram amostras de tamanho semelhante. Exame inicial dos dados mostrou que embora houvesse certa heterogeneidade na distribuição dos escores entre os grupos do presente estudo, os testes propostos são suficientemente robustos para lidar com a variância encontrada. A análise multivariada 2 (mães com indicadores de depressão versus mães sem indicadores de depressão) x 2 (comportamentos facilitadores versus comportamentos não-facilitadores) utilizando os escores totais dos comportamentos maternos revelou um efeito significativo da presença de indicadores de depressão da mãe na incidência total dos comportamentos maternos facilitadores e nãofacilitadores $(F(2,23)=6,65, \rho<0,006$; Wilk's lambda $=$ $0,63)$. Os resultados indicaram que mães com indicadores de depressão apresentaram menos comportamentos facilitadores $(p<0,001)$ e uma tendência a apresentar mais comportamentos não-facilitadores $(p<0,07)$ quando comparadas às mães sem indicadores de depressão. 
A segunda análise multivariada 2 (mães com indicadores de depressão versus mães sem indicadores de depressão) x 2 (afeto positivo versus afeto negativo) realizada nos escores totais dos comportamentos infantis revelou um efeito significativo da presença de indicadores de depressão materna na incidência total de afeto infantil positivo e negativo $(F(2,23)$ $=3,89, \rho<0,03$; Wilk's lambda $=0,74)$. Os resultados indicaram que os bebês de mães com indicadores de depressão apresentaram mais afeto negativo $(p<0,01)$ do que bebês de mães sem indicadores de depressão. Não houve diferença significativa para o total de comportamentos positivos dos bebês de ambos os grupos.

Para examinar mais detalhadamente o efeito da presença de indicadores de depressão materna sobre cada categoria de comportamentos materno e infantil, análise de variância foi realizada separadamente para cada categoria de comportamentos. A Tabela 4 apresenta a incidência média, desvio padrão, valor de $F$ e nível de significância para cada categoria de comportamentos maternos. Os resultados revelaram algumas diferenças significativas entre os grupos na incidência dos comportamentos observados. As mães com indicadores de depressão, quando comparadas com mães do outro grupo, apresentaram uma incidência de comportamentos significativamente menor nas categorias mantém atenção em um brinquedo $(\rho<0,03)$, demonstra ternura e afeição $(\rho<0,02)$ e uma incidência significativamente maior na categoria demonstra apatia $(\rho<0,03)$. Além disso, há uma tendência das mães com indicadores de depressão a apresentarem menor incidência na categoria introduz um brinquedo $(\rho<0,08)$.

A Tabela 5 apresenta a incidência média, desvio padrão, valor de $F$ e nível de significância para cada categoria de comportamentos infantis. Os bebês de mães com indicadores de depressão apresentaram uma incidência significativamente maior na categoria vocaliza negativamente $(\rho<0,01)$. Além disso, ocorreu uma diferença marginalmente significativa na categoria sorri $(\rho<0,06)$, indicando que os bebês de mães com indicadores de depressão tenderam a sorrir menos do que os de mães sem indicadores de depressão.

Tabela 3. Incidência média, desvio padrão, valor de F e nível de significância para o total de escores de comportamentos maternos e infantis nos grupos com e sem indicadores de depressão

\begin{tabular}{lccc}
\hline \multicolumn{1}{c}{ Comportamentos } & $\begin{array}{c}\text { Mães com indicadores de depressão } \\
M(D P)\end{array}$ & $\begin{array}{c}\text { Mães sem indicadores de depressão } \\
M(D P)\end{array}$ & F \\
\hline Maternos & & & \\
Facilitadores & $37,45(8,66)$ & $46,73(3,84)$ & 13,69 \\
Não-facilitadores & $17,90(9,40)$ & $12,00(6,65)$ & 0,001 \\
Infantis & & & 3,53 \\
Afeto positivo & $29,90(7,75)$ & $33,33(5,55)$ & \\
Afeto negativo & $4,54(2,80)$ & $2,13(1,84)$ & 1,73 \\
\hline
\end{tabular}

Tabela 4. Incidência média, desvio padrão e nível de significância para cada categoria de comportamentos maternos nos grupos com e sem indicadores de depressão

\begin{tabular}{lrrr}
\hline \multicolumn{1}{c}{ Comportamentos maternos } & Mães com indicadores de depressão & Mães sem indicadores de depressão & \multicolumn{2}{c}{ F } & $\rho<$ \\
\hline Introduz um brinquedo & $M(D P)$ & $2,07(1,87)$ & 3,30 \\
Mantém a atenção & $0,91(1,14)$ & $19,47(2,59)$ & 0,08 \\
Expressa prazer e alegria & $16,36(4,34)$ & $2,20(2,11)$ & 5,19 \\
Demonstra ternura e afeição & $0,91(1,64)$ & $23,00(2,30)$ & 0,03 \\
Redireciona a atenção & $19,27(5,46)$ & $5,29(3,14)$ & 0,10 \\
Evidencia intrusividade & $6,00(3,92)$ & $4,27(3,20)$ & 5,64 \\
Demonstra apatia & $4,82(3,74)$ & $0,33(1,05)$ & 0,34 \\
Demonstra contrariedade & $3,64(5,63)$ & 0,56 & 0,16 \\
\hline
\end{tabular}

Tabela 5. Incidência média, desvio padrão e nível de significância para cada categoria de comportamentos infantis nos grupos com e sem indicadores de depressão

\begin{tabular}{|c|c|c|c|c|}
\hline Comportamentos infantis & $\begin{array}{l}\text { Mães com indicadores de depressão } \\
\qquad M(D P)\end{array}$ & $\begin{array}{l}\text { Mães sem indicadores de depressão } \\
\qquad M(D P)\end{array}$ & $\mathbf{F}$ & $\rho<$ \\
\hline Focaliza atenção em um brinquedo & $18,18(3,76)$ & $19,27(3,77)$ & 0,52 & 0,47 \\
\hline Sorri & $0,45(0,30)$ & $1,20(1,90)$ & 3,65 & 0,06 \\
\hline Vocaliza positivamente & $10,45(6,59)$ & $12,40(4,79)$ & 0,76 & 0,39 \\
\hline Busca proximidade & $1,18(1,89)$ & $0,47(0,92)$ & 1,64 & 0,21 \\
\hline Rejeita brinquedo & $1,91(1,76)$ & $1,20(1,42)$ & 1,29 & 0,26 \\
\hline Chora & $0,18(0,60)$ & $0,33(0,62)$ & 0,39 & 0,53 \\
\hline Vocaliza negativamente & $2,27(2,15)$ & $0,47(0,83)$ & 8,88 & 0,01 \\
\hline Afasta-se/resiste ao contato & $0,18(0,40)$ & $0,13(0,35)$ & 0,10 & 0,74 \\
\hline
\end{tabular}




\section{Discussão}

Os resultados do presente estudo apóiam as expectativas iniciais de que mães com e sem indicadores de depressão apresentariam uma incidência de comportamentos diferente durante a situação de interação livre. As mães com indicadores de depressão apresentaram significativamente menos comportamentos facilitadores da exploração de objetos pelo bebê e também uma tendência a apresentar mais comportamentos não-facilitadores dessa exploração. Juntos, esses achados apóiam a expectativa de que a interação mãebebê parece ser influenciada pela presença da depressão materna.

Uma análise específica de cada categoria de comportamentos maternos também indicou diferenças significativas entre os grupos em diversas categorias. Mães com indicadores de depressão apresentaram significativamente menos comportamentos de manutenção da atenção de seus bebês em um brinquedo, além de uma tendência a introduzirem menos brinquedos para a criança. Esses resultados apóiam as evidências de que mães deprimidas tendem a ser menos atentas em relação aos brinquedos do bebê (Breznitz \& Friedman, 1988). Por outro lado, contrariam os resultados encontrados por Hart e cols. (1998) em um estudo que observou a interação mãebebê, o qual não revelou diferenças no uso dessas categorias entre mães deprimidas e não-deprimidas. É possível que essa inconsistência entre os estudos deva-se a problemas metodológicos, em virtude de que, no presente estudo, utilizou-se um tempo maior de observação da interação do que no estudo de Hart e cols.(1998) baseado em apenas três minutos de interação. Poder-se-ia pensar que o período mais prolongado de observação tenha atenuado a artificialidade da sessão de observação, permitindo às mães a manifestação de comportamentos mais condizentes com aqueles que eventualmente apresentariam em situações naturais de interação com seus bebês. Nesse sentido, a exacerbação das diferenças entre os grupos que ocorreu no presente estudo poderia ser explicada em termos de uma gradativa descontração das mães frente à situação de filmagem.

Outra diferença importante entre os grupos refere-se às evidências de que mães com indicadores de depressão foram menos enfáticas na demonstração física e verbal de afeto e mais apáticas do que mães sem indicadores de depressão. Esses resultados apóiam aqueles encontrados por RadkeYarrow e cols. (1993) que, ao examinarem os escores totais da expressão de afeto de mães deprimidas em interação com seus bebês, verificaram que estas demonstraram mais afeto negativo do que mães não-deprimidas. Mais especificamente, os autores encontraram que mães deprimidas mostraram mais expressões de ansiedade, tristeza, apatia e menos ternura e afeição com os bebês do que mães não-deprimidas.

Embora as diversas diferenças reveladas entre os grupos no presente estudo sejam apoiadas pela literatura, outras que eram esperadas, como, por exemplo, que mães com indicadores de depressão apresentariam maior intrusividade e redirecionamento da atenção do bebê durante a interação (Breznitz \& Friedman, 1988; Hart \& cols., 1998; Hart \& cols., 1999; McElwain \& Volling, 1999) não foram encontradas. Além disso, alguns autores consideraram que demonstrações de contrariedade e expressões de prazer e alegria também constituiriam dimensões afetivas relevantes para a identificação de diferenças comportamentais ocasionadas pela depressão da mãe (Teti \& Gelfand, 1991). Porém, no presente estudo, não foram encontradas diferenças significativas entre os grupos em relação a essas categorias.

Quanto às categorias de comportamentos infantis, diferenças também foram encontradas entre os grupos. Os resultados da análise dos escores totais dos comportamentos infantis revelaram que bebês de mães com indicadores de depressão demonstraram mais afeto negativo do que bebês de mães do outro grupo. Esses achados apóiam evidências anteriores sobre mais demonstração de afeto negativo entre bebês de mães deprimidas, independentemente do gênero da criança (Radke-Yarrow \& cols., 1993). Porém, corroboram apenas parcialmente aqueles encontrados por Hart e cols. (1998) que encontraram que filhas de mães deprimidas apresentaram mais afeto negativo, não ocorrendo o mesmo com bebês do sexo masculino. Além disso, os autores encontraram que filhas de mães deprimidas apresentaram também menos afeto positivo. No presente estudo, não foram encontradas diferenças entre os bebês de mães com e sem indicadores de depressão em relação aos escores totais de afeto positivo. Da mesma forma, não foi encontrado efeito do gênero do bebê na incidência total de comportamentos maternos e infantis.

Já os resultados das análises realizadas separadamente para cada uma das categorias de comportamentos infantis revelaram diferenças expressivas em apenas duas das oito categorias examinadas. Bebês de mães com indicadores de depressão apresentaram significativamente mais vocalizações negativas. Além disso, foi encontrada uma diferença marginalmente significativa na categoria sorri, indicando que os bebês de mães com indicadores de depressão sorriam menos do que bebês de mães sem indicadores de depressão. Resultados semelhantes foram encontrados por Field e cols. (1996), ao compararem o engajamento de bebês de 12 meses, filhos de mães com e sem depressão, em uma situação estruturada de exploração de brinquedos. Eles encontraram que bebês de mães deprimidas sorriam menos e tinham mais vocalizações negativas.

Afora as diferenças relatadas acima que são apoiadas pela literatura, alguns estudos têm indicado que bebês de mães deprimidas tendem a focalizar menos a atenção em brinquedos durante a interação com suas mães (Hart \& cols., 1998), rejeitam brinquedos mais freqüentemente (Hart \& cols., 1998; Seiner \& Gelfand, 1995), têm menos vocalizações positivas (Field \& cols., 1996), afastam-se das mães e choram mais vezes (Radke-Yarrow \& cols., 1993). Porém, no presente estudo não foram constatadas diferenças significativas entre os grupos no que se refere a esses comportamentos.

Quanto à ausência de diferenças entre os grupos em algumas categorias de comportamentos maternos e infantis, algumas considerações podem ser feitas. Inicialmente, poder-se-ia pensar que a ausência de diferenças em relação às categorias maternas evidencia intrusividade e redireciona $a$ atenção poderia estar relacionada a maior manifestação de apatia por parte das mães com indicadores de depressão. Uma vez estando mais apáticas durante a interação, as mães agiriam com menos intrusividade e mais passivamente diante da possibilidade de estimular o bebê com vários brinquedos. $\mathrm{Na}$ verdade, a literatura aponta para dois tipos de estilo 
comportamental que uma mãe deprimida pode manifestar ao interagir com o seu bebê. O primeiro caracteriza-se pelo afastamento emocional da mãe enquanto o segundo caracteriza-se por sua intrusividade e excesso de estimulação (Hart \& cols., 1999; McElwain \& Volling, 1999). Embora a avaliação desses estilos comportamentais não tenha sido privilegiada no presente estudo, os resultados encontrados permitem pensar em uma tendência das mães com indicadores de depressão a manifestarem um estilo mais apático do que intrusivo ao interagirem com seus bebês.

Poder-se-ia pensar também que a ausência de diferenças em relação à incidência de comportamentos maternos intrusivos e de redirecionamento da atenção do bebê esteja associada à própria sessão de observação. No presente estudo optou-se pela observação de uma situação de brinquedo livre ao invés de uma situação estruturada, como a que foi utilizada por McElwain e Volling (1999) ao avaliarem a intrusividade de mães deprimidas e não-deprimidas com seus bebês. Esses autores apontaram que sessões de brinquedo previamente estruturadas, nas quais propõe-se à mãe e ao bebê a resolução de uma determinada tarefa, requerem uma maior atividade e direcionamento por parte da mãe, o que pode ter exacerbado o aparecimento de comportamentos intrusivos entre as mães com depressão. No presente estudo, a ausência de uma atividade estruturada para a díade realizar pode ter contribuído para que esses comportamentos não pudessem ser vistos como mais característicos de um ou outro grupo de mães. Em virtude disso, a situação de brinquedo livre utilizada neste estudo pode ter contribuído para a ausência de diferenças significativas em algumas categorias de comportamentos infantis, como por exemplo focaliza a atenção, ao não exigir da mãe uma participação mais efetiva. Alguns autores sugeriram que os comportamentos dos bebês na exploração de brinquedos são diretamente influenciados pelas estratégias de atenção utilizadas pela mãe (Lawson \& cols., 1992). Apoiando essas concepções, a literatura aponta também que bebês de mães deprimidas tendem a focalizar menos a atenção nos objetos à sua volta, enquanto a mãe tende a redirecionar a atenção do bebê e a ser mais intrusiva (Breznitz \& Friedman, 1988; Hart \& cols., 1998).

A ausência de diferenças significativas entre os grupos em relação às categorias de comportamentos maternos $d e$ monstra contrariedade e expressa prazer e alegria corrobora o estudo de Radke-Yarrow e cols. (1993), que também não encontraram diferenças significativas entre mães deprimidas e não-deprimidas nessas categorias, embora tivessem expectativas nesse sentido. Poder-se-ia pensar que essa ausência de diferenças no presente estudo esteja relacionada a possíveis esforços das mães no sentido de estarem emocionalmente mais próximas de seus bebês, cantando, gargalhando e comemorando seus feitos tanto quanto mães que não apresentavam indicadores de depressão. Como sugeriu Stern (1997), uma vez deprimidas as mães lutariam contra sua relativa falta de disponibilidade com o bebê e, por vezes, obteriam sucesso nessas tentativas.

$\mathrm{O}$ fato de que poucas mães do presente estudo apresentavam níveis mais graves de depressão pode ter contribuído para a ausência de diferenças entre os grupos tanto nas categorias de comportamentos maternos como infantis. É possível pensar que as diferenças entre os grupos de mães com e sem indicadores de depressão pudessem ser exacerbadas diante de um número maior de mães com depressão em níveis mais graves. Além disso, alguns autores sugeriram que as repercussões da depressão da mãe na interação mãe-bebê estão estreitamente relacionadas ao tempo de permanência do diagnóstico, no sentido de que, quanto maior esse tempo, maiores seriam as implicações nos comportamentos maternos e infantis (Campbell \& cols.1995; Field \& cols., 1996). No presente estudo, a cronicidade dos quadros depressivos apresentados pelas mães não foi examinada. Apesar da amostra ser derivada de uma investigação longitudinal que acompanhava as mães desde a gestação, foram investigados indicadores de depressão materna apenas quando os bebês estavam com 12 meses de vida.

Um outro ponto a ser ressaltado em relação à ausência de diferenças entre os grupos em algumas categorias de comportamentos da mãe e do bebê, refere-se à adequação do protocolo utilizado no presente estudo para o exame da interação mãe-bebê. É possível que ele não tenha sido suficientemente sensível para captar algumas particularidades na dinâmica mãe-bebê dos dois grupos, principalmente em virtude do tempo reduzido da sessão de observação (seis minutos). Poder-se-ia pensar que durante o curto período de filmagem as mães tenham se preocupado em mostrar o melhor de si, o que colaboraria para a ausência de diferenças significativas entre os grupos em algumas categorias como, por exemplo, na categoria materna demonstra contrariedade e na categoria infantil afasta-se/resiste ao contato. Essa possibilidade parece ter sido respaldada no presente estudo pelas evidências da ocorrência de algumas diferenças que não foram encontradas em um estudo semelhante, no qual os autores utilizaram um período de tempo ainda mais reduzido na observação da interação mãe-bebê (Hart \& cols., 1998).

Tendo em vista essas considerações, é importante que novos estudos busquem ampliar a compreensão sobre as implicações da depressão materna na qualidade de interação mãe-bebê, utilizando amostras maiores investigadas em situações estruturadas e também naturalísticas, permitindo que se compreenda a dinâmica da interação em diferentes contextos. De qualquer modo, os resultados do presente estudo reafirmam o impacto potencial da depressão materna para a interação mãe-bebê. Apontam particularmente para o seu papel no final primeiro ano de vida, considerando-se que muitos autores têm se dedicado principalmente ao estudo de suas repercussões nos primeiros meses após o parto.

\section{Referências}

Beck, A. T. \& Steer, R. A. (1993). Beck Depression Inventory. Manual. San Antonio: Psychological Corporation.

Beeghly, M., Weinberg, M. K., Olson, K. L., Kernan, H., Riley, J. \& Tronick, E. Z. (2002). Stability and change in level of maternal depressive symptomatology during the first postpartum year. Journal of Affective Disorders, 71(1-3), 169-180.

Breznitz, Z. \& Friedman, S. L. (1988). Toddler's concentration: Does maternal depression make a difference? Journal of Child Psychology and Psychiatry, 29(3), 267-279.

Brown, S., Lumley, J., Small, R. \& Astbury, J. (1994). Missing voices: The experience of motherhood. New York: Oxford University Press. 
Campbell, S. B. \& Cohn, J. F. (1997). Prevalence and correlates of postpartum depression in first-time mothers. Journal of Abnormal Psychology, 100(4), 594-599.

Campbell, S. B., Cohn, J. F. \& Meyers, T. (1995). Depression in first-time mothers: Mother-infant interaction and depression chronicity. Developmental Psychology, 31(3), 349-357.

Cooper, P. J., Campbell, E. A., Day, A., Kennerley, H. \& Bond, A. (1988). Non-psychotic psychiatric disorder after childbirth: A prospective study of prevalence, incidence, course and nature. British Journal of Psychiatry, 152, 799-806.

Cox, J. L., Murray, D. \& Chapman, G. (1993). A controlled study of the onset, duration and prevalence of postnatal depression. British Journal of Psychiatry, 163, 27-31.

Cummings, M. E. \& Davies, P. T. (1994). Maternal depression and child development. Journal of Child Psychology and Psychiatry, 35(1), 73-112.

Cunha, J. A. (2001). Escalas Beck. São Paulo: Casa do Psicólogo.

Cunha, J. A., Prieb, R. G. G., Goulart, P. M. \& Lemes, R. B. (1996). O uso do inventário de Beck para avaliar depressão em universitários. Psico, 27(1), 107-115.

Dodge, K. A. (1990). Developmental psychopathology in children of depressed mothers. Developmental Psychology, 26(1), 3-6.

Field, T. (1995). Infants of depressed mothers. Infant Behavior and Development, 18, 1-13.

Field, T. (1998). Maternal depression effects on infants and early interventions. Preventive Medicine, 27, 200-203.

Field, T., Estroff, D. B., Yando, R., Del Valle, C., Malphurs, J. \& Hart, S. (1996). Depressed mother's perceptions of infant vulnerability are related to later development. Child Psychiatry and Human Development, 27(1), 43-53.

Hart, S., Field, T., Del Valle, C. \& Pelaez-Nogueras, M. (1998). Depressed mother's interactions with their one-year-old infants. Infant Behavior and Development, 21(3), 519-525.

Hart, S., Jones, N. A., Field, T. \& Lundy, B. (1999). One-year-old infants of intrusive and withdraw depressed mothers. Child Psychiatry and Human Development, 30(2), 111-120.

Hossain, Z., Field, T., Gonzalez, J., Malphurs, J. \& Del Valle, C. (1994). Infants of depressed mothers interact better with their nondepressed fathers. Infant Mental Health Journal, 15(4), 348-357.

Klaus, M. H., Kennell, J. H. \& Klaus, P. (2000). Vínculo: Construindo as bases para um apego seguro e para a independência. Porto Alegre: Artes Médicas.

Lawson, R., Parrinello, R. \& Ruff, H. (1992). Maternal behavior and infant attention. Infant Behavior and Development, 15(2), 209-229.

McElwain, N. L. \& Volling, B. (1999). Depressed mood and marital conflict: Relations to maternal and paternal intrusiveness with one-year-old infants. Journal of Applied Developmental Psychology, 20(1), 63-83.

Murray, D., Cox, J., Chapman, G. \& Jones, P. (1995). Childbirth: Life event or start of a long-term difficulty? British Journal of Psychiatry, 166, 595-600.

Nachmias, C. \& Nachmias, D. (1996). Research methods in the social sciences. London: Arnolds.

O’Hara, M. W., Neunaber, D. J. \& Zekoski, E. M. (1984). Prospective study of postpartum depression: Prevalence, course, and predictive factors. Journal of Abnormal Psychology, 93, 158-171.
Pelaez-Nogueras, M., Field, T., Cigales, M., Gonzalez, A. \& Clasky, S. (1994). Infants of depressed mothers show less depressed behavior with their nursery teachers. Infant Mental Health Journal, 15(4), 358-367.

Piccinini, C. A., Tudge, J., Lopes, R. S. \& Sperb, T. M. (1998). Estudo longitudinal de Porto Alegre: Da gestação à escola. Projeto de Pesquisa, Universidade Federal do Rio Grande do Sul, Porto Alegre.

Radke-Yarrow, M., McCann, K., DeMulder, E., Belmont, B., Martinez, P. \& Richardson, D. (1995). Attachment in the context of high-risk conditions. Development and Psychopathology, $7(2), 247-265$.

Radke-Yarrow, M., Nottelmann, E., Belmont, B. \& Welsh, J. D. (1993). Affective interactions of depressed and nondepressed mothers and their children. Journal of Abnormal Child Psychology, 21(6), 683-695.

Robson, C. (1995). Real word research: A resource for social scientists and practiotioner-researchs. Oxford, UK: Blackwell.

Schwengber, D. D. S. \& Piccinini, C. A. (2001). Protocolo de análise da interação mãe-bebê de um ano de idade durante a interação livre. Trabalho não publicado, Universidade Federal do Rio Grande do Sul, Porto Alegre.

Seiner, S. H. \& Gelfand, D. M. (1995). Effects of mother's simulated withdrawal and depressed affect on mother-toddler interactions. Child Development, 66(5), 1519-1528.

Stern, D. N. (1997). A constelação da maternidade: O panorama da psicoterapia pais/bebê. Porto Alegre: Artes Médicas.

Tamis-LeMonda, C. \& Bornstein, M. (1989). Habituation and maternal encouragement of attention in infancy as predictors of toddler language, play and representational competence. Child Development, 60(3), 738-751.

Teti, D. M. \& Gelfand, D. M. (1991). Behavioural competence among mothers of infants in the first year: The mediational role of maternal self-efficacy. Child Development, 62(5), 919-929.

Tronick, E. Z. \& Weinberg, M. K. (1997). Depressed mothers and infants: Failure to form dyadic states of consciousness. Em L. Murray \& P. J. Cooper (Orgs.), Postpartum Depression and Child Development (pp. 54-81). Guilford Press, New York. 